\title{
Hemoglobin S-nitrosylation plays an essential role in cardioprotection
}

\author{
Rongli Zhang, ${ }^{1,2}$ Douglas T. Hess, ${ }^{1,2}$ James D. Reynolds, ${ }^{1,3,4}$ and Jonathan S. Stamler ${ }^{1,2,4}$ \\ IInstitute for Transformative Molecular Medicine, Case Western Reserve University School of Medicine and University Hospitals Cleveland Medical Center, Cleveland, Ohio, USA. ${ }^{2}$ Department of Medicine, \\ Case Western Reserve University School of Medicine, Cleveland, Ohio, USA. ${ }^{3}$ Department of Anesthesiology and Perioperative Medicine, Case Western Reserve University School of Medicine, Cleveland, Ohio, \\ USA. ${ }^{4}$ Harrington Discovery Institute, University Hospitals Cleveland Medical Center, Cleveland, Ohio, USA.
}

\begin{abstract}
Homeostatic control of tissue oxygenation is achieved largely through changes in blood flow that are regulated by the classic physiological response of hypoxic vasodilation. The role of nitric oxide (NO) in the control of blood flow is a central tenet of cardiovascular biology. However, extensive evidence now indicates that hypoxic vasodilation entails S-nitrosothiol-based (SNO-based) vasoactivity (rather than NO per se) and that this activity is conveyed substantially by the $\beta$ Cys93 residue in hemoglobin. Thus, tissue oxygenation in the respiratory cycle is dependent on S-nitrosohemoglobin. This perspective predicts that red blood cells (RBCs) may play an important but previously undescribed role in cardioprotection. Here, we have found that cardiac injury and mortality in models of myocardial infarction and heart failure were greatly enhanced in mice lacking BCys93 S-nitrosylation. In addition, $\beta$ Cys93 mutant mice exhibited adaptive collateralization of cardiac vasculature that mitigated ischemic injury and predicted outcomes after myocardial infarction. Enhanced myopathic injury and mortality across different etiologies in the absence of $\beta$ Cys93 confirm the central cardiovascular role of RBC-derived SNO-based vasoactivity and point to a potential locus of therapeutic intervention. Our findings also suggest the possibility that RBCs may play a previously unappreciated role in heart disease.
\end{abstract}

\section{Introduction}

Control of blood flow in the microvasculature (i.e., tissue perfusion) in response to altered $\mathrm{O}_{2}$ consumption is governed by an autoregulatory mechanism whereby flow is coupled to tissue $\mathrm{pO}_{2}$ (1). It has been shown that red blood cells (RBCs) can themselves provide the signal that couples flow to $\mathrm{pO}_{2}$, and it was proposed that this signal is mediated by the $\mathrm{pO}_{2}$-regulated release of nitric oxide-derived (NO-derived) vasodilatory activity that is conveyed by hemoglobin $(\mathrm{Hb}) S$-nitrosylated at Cys93 of the $\beta$-chain $(\mathrm{SNO}-\mathrm{Hb})(2,3)$ (reviewed in ref. 4$)$. This proposal was recently confirmed (5) by analyses of engineered mice in which the critical Cys that mediates vasodilation by $\mathrm{Hb}$ was replaced by Ala ( $\beta$ Cys93Ala) (6). In the absence of $\beta$ Cys93, peripheral blood flow and tissue oxygenation are markedly decreased at normoxia and decline excessively during hypoxia (5). In addition, the $\beta$ Cys93Ala mutation results in myocardial ischemia under basal, normoxic conditions and in acute cardiac decompensation and enhanced mortality during transient hypoxia (5). Therefore, the ability of mammals to oxygenate tissues, including oxygenation of the heart required for normal myocardial function, is evidently dependent in large part upon SNO-Hb. Tissue oxygenation is thus governed not only by the $\mathrm{O}_{2}$ content, but also by the SNO

\section{Related Commentary: p. 4402}

Conflict of interest: J.S. Stamler has financial interests in Nivalis Therapeutics Inc., Adamas Pharmaceuticals, and LifeHealth. J.D. Reynolds has a financial interest in Miach Medical Innovations. J.S. Stamler and J.D. Reynolds have licensed technology to Novartis Submitted: August 30, 2016; Accepted: October 6, 2016

Reference information: / Clin Invest. 2016;126(12):4654-4658. doi:10.1172/JCI90425. content of $\mathrm{Hb}$, consistent with the strict evolutionary conservation of $\beta$ Cys93 in all mammals (7).

The most common causes of morbidity and mortality in Western cultures are cardiovascular in origin. Both extent of injury and outcomes in the most prevalent cardiac diseases, i.e., acute myocardial infarction (MI) and heart failure, reflect uncoupling of blood flow from metabolic demand $(8,9)$. Our recent findings in $\beta$ Cys93Ala animals (5) support a new perspective in which RBCs subserve blood-flow regulation previously ascribed to the endothelium. To test the resulting prediction that regulation of blood flow by RBCs would play a role in response to cardiac insult, we extended our analysis of $\beta$ Cys93Ala animals to well-established experimental murine models of MI, induced by ischemia-reperfusion (IR) injury (10), and pressure overloadinduced cardiomyopathy, induced by transaortic constriction (TAC) (11). Our results demonstrate that $\beta$ Cys93 in Hb plays an essential role in the outcome of both insults.

\section{Results and Discussion}

The mice employed comprised 3 genetically engineered strains expressing "humanized" $\mathrm{RBC} \mathrm{Hb}(6)$, which we designated $\gamma \beta \mathrm{C} 93$ (control mice), $\gamma \beta \mathrm{C} 93 \mathrm{~A}$, and $\beta \mathrm{C} 93 \mathrm{~A}$. In all engineered strains, the murine $\alpha$ and $\beta$ subunits of RBC Hb were replaced with the human versions. In $\gamma \beta \mathrm{C} 93$ and $\gamma \beta \mathrm{C} 93 \mathrm{~A}$ mice (genetically matched with the exception of $\beta$ Cys93), RBCs also contained the human $\gamma$ (fetal) subunit; in all 3 strains, RBCs retained the murine $\gamma$ subunit, but $\gamma$ subunits remained minor species $(5,12)$. $\beta C y$ s 93 was replaced with Ala in $\beta$ C93A and $\gamma \beta$ C93A mice. It has previously been shown that total $\mathrm{Hb}$ concentration, blood $\mathrm{O}_{2}$ content, $\mathrm{O}_{2}$-binding curves, blood pressure, and heart rate do not differ significantly, or meaningfully, 
A

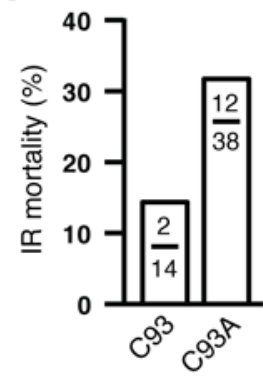

B

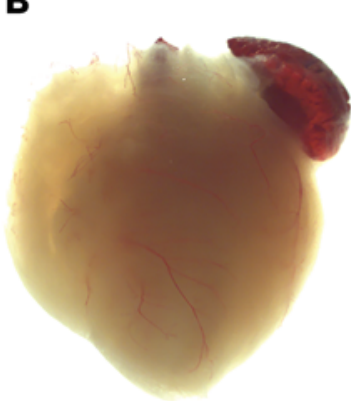

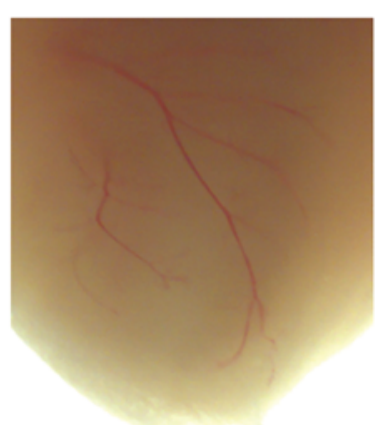

C

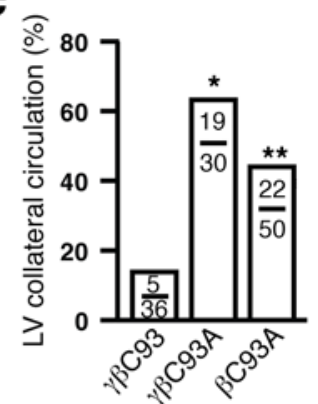

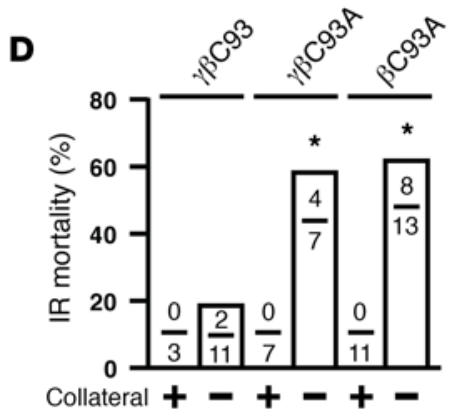

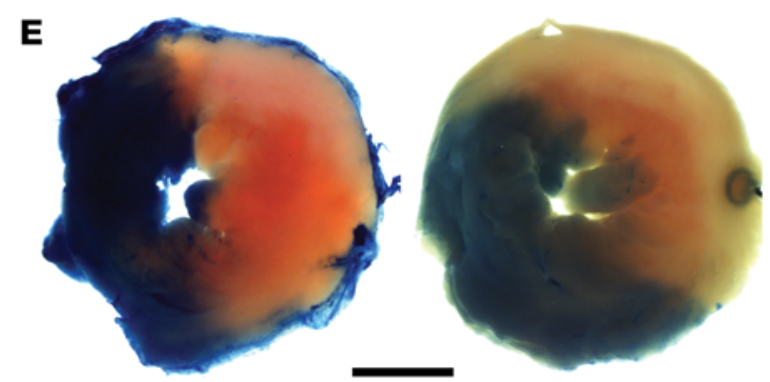

$\mathbf{F}$

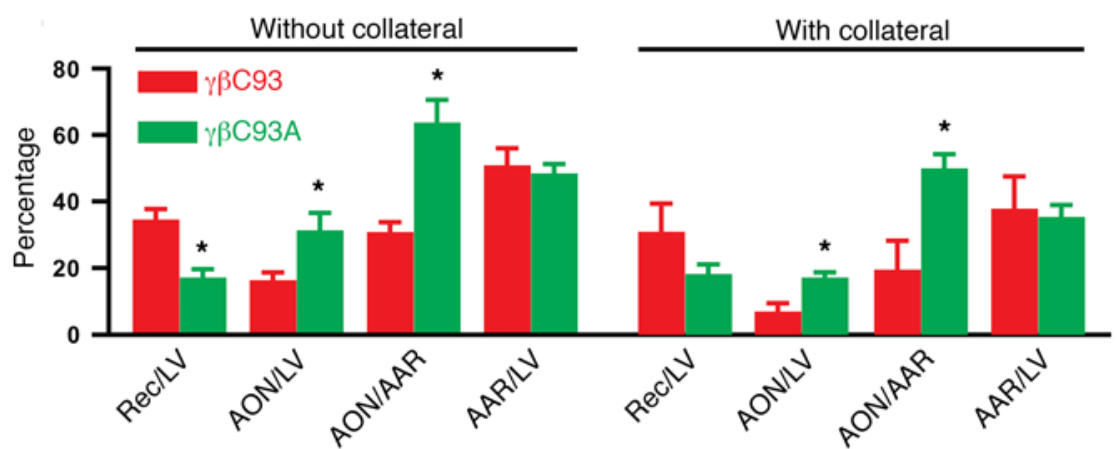

G

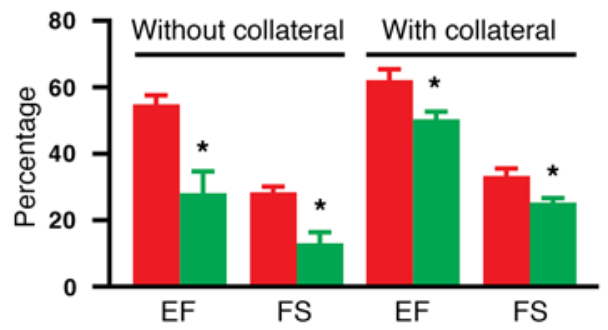

H

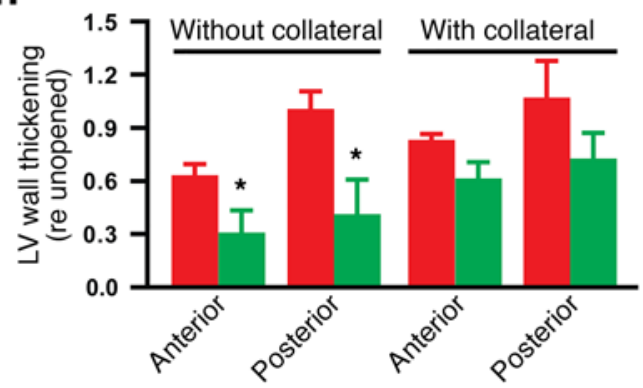

Figure 1. Enhanced MI-induced cardiac injury and mortality in the absence of $\beta$ Cys93. (A) IR-induced mortality is greatly enhanced in mice lacking $\beta$ Cys93 (C93A $=\gamma \beta$ C93A and $\beta$ C93A) versus control mice $(C 93=\gamma \beta C 93)$. In panels $\mathbf{A}, \mathbf{C}$, and $\mathbf{D}$, numbers within histograms specify absolute ratios for each observation (B) Representative angiography illustrating collateralized circulation of the LV free wall to supply the apex in a mutant $\beta C 93 A$ mouse. Lower (left; $\times 6.7$ ) and higher (right; $\times 15.8$ ) magnification views are shown. (C) Collateralization supplying the LV is significantly more likely in $\gamma \beta C 93 A$ and $\beta C 93 A$ versus $\gamma \beta$ C93 mice. ${ }^{*} P<0.0001$ for $\gamma \beta$ C93 versus $\gamma \beta$ C93A and ${ }^{* *} P=0.0043$ for $\gamma \beta$ C93 versus $\beta$ C93A, respectively, by Fisher's exact test (2-sided). The difference in collateralization between $\gamma \beta$ C93A and $\beta$ C93A mice was not significant $(P>0.05)$. (D) Mortality during 24 hours of IR is determined by the presence versus absence of collateral circulatory supply of the $L V$ and is enhanced in $\gamma \beta C 93 A$ and $\beta$ C93A versus $\gamma \beta C 93$ mice. ${ }^{*} P<0.0001$ vs. collateral by Fisher's exact test (2-sided). (E) Representative Evans blue/TTC-stained LV slices illustrating areas of recovery (red) and necrosis (white) in a $\gamma \beta C 93$ mouse (left) and in a $\gamma \beta$ C93A mouse (right). Scale bar: $2 \mathrm{~mm}$. (F) In $\gamma \beta C 93 \mathrm{~A}$ versus $\gamma \beta C 93$ control mice surviving 24 hours after IR, the area of recovery (Rec/LV; LV, total LV area) is significantly diminished and the area of necrosis (AON/LV) and the ratio of area of necrosis/area at risk (AON/AAR) are significantly enhanced, and enhanced injury was evident in the absence of $\beta C 93$ even when collateralization was present. (C) Echocardiography of surviving mice reveals that $L V$ ejection fraction (EF) and fractional shortening (FS) are significantly diminished in $\gamma \beta C 93 \mathrm{~A}$ versus $\gamma \beta C 93$ control mice, and these differences were evident even when collateralization was present. $(\mathbf{H})$ Thickening of the LV anterior and posterior walls in the absence of collateral blood supply is significantly diminished in $\gamma \beta$ C93A versus $\gamma \beta$ C93 mice. In F-H, $n=3-7$ for mice with collateral blood supply and $n=3-9$ for mice without collateral blood supply. ${ }^{*} P<0.05$ vs. $\gamma \beta C 93$ by Student's $t$ test (2-tailed). 


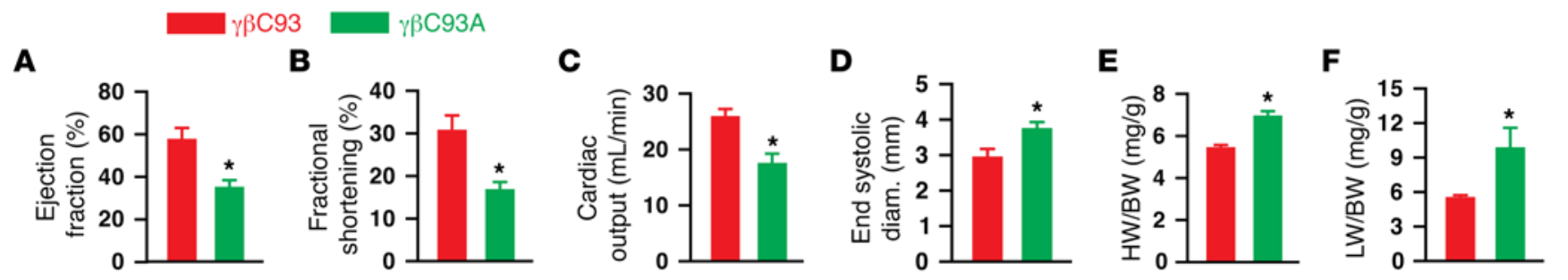

Figure 2. Assessment of acute effects of pressure overload (TAC) on cardiac function in C93A mutant animals. $\gamma \beta$ C93 and $\gamma \beta C 93 A$ mice were compared at 2 days. (A-C) Echocardiography revealed significantly decreased ejection fraction (A), fractional shortening (B), and cardiac output (C) in $\gamma \beta C 93 A$ versus $\gamma \beta$ C93 mice. (D) End systolic diameter (end systolic diam.) was significantly greater in $\gamma \beta$ C93A versus $\gamma \beta C 93$ mice. (E and F) The ratios of heart weight with respect to body weight (HW/BW) (E) and lung weight with respect to body weight (LW/BW) (F) were significantly increased in $\gamma \beta C 93 A$ versus $\gamma \beta C 93$ mice. $n=5-7 .{ }^{*} P<0.05$ vs. $\gamma \beta C 93$ by Student's $t$ test (2-tailed).

among these strains under basal conditions $(5,6,12)$. We focused our analysis on comparisons between the $\gamma \beta C 93$ and $\gamma \beta C 93 \mathrm{~A}$ strains, isogenic except for the mutation of $\beta C 93$. However, we have shown that the cardiovascular phenotypes of $\gamma \beta \mathrm{C} 93$ or $\beta \mathrm{C} 93 \mathrm{~A}$ (versus $\gamma \beta C$ C93) mice are similar (5), and because of difficulties with breeding and therefore maintaining colonies of sufficient numbers for studies (5), we included comparisons with $\beta$ C93A mice where those comparisons help clarify interpretations of results.

MI was induced by occlusion of the left coronary artery (LCA) for 30 minutes, followed by reperfusion for 24 hours (IR). All mice recovered from the surgery, but within 24 hours of IR, approximately $14 \%(2 / 14)$ of control mice died, whereas approximately $32 \%$ (12/38) of mice lacking $\beta C 93$ died (Figure 1A). Thus, delivery of vasodilatory activity via $\beta C 93$ is cardioprotective in the setting of IR-induced MI.

Employing angiography or Evans blue staining (see Supplemental Methods; supplemental material available online with this article; doi:10.1172/JCI90425DS1), we observed that major collaterals of the LCA or of the right coronary artery (RCA) traversed the left ventricle (LV) to terminate in the apex (Figure 1B) in $14 \%$ $(5 / 36)$ of $\gamma \beta C 93$ control mice (Figure 1C) and that collateralization was greatly enhanced in $\gamma \beta C 93 \mathrm{~A}(63 \% ; 19 / 30)$ and $\beta \mathrm{C} 93 \mathrm{~A}$ $(44 \% ; 22 / 50)$ mutant mice (Figure $1 \mathrm{C})$. The incidence of collateralization did not differ significantly between $\gamma \beta C 93 \mathrm{~A}$ and $\beta C 93 \mathrm{~A}$ mice (Figure 1C). Collateralization of the LV from the LCA versus the RCA, which did not occur together, was equally likely in all strains. Enhanced collateralization in $\gamma \beta \mathrm{C} 93 \mathrm{~A}$ and $\beta$ C93A mice was consistent with compensation for inadequate myocardial oxygenation in the absence of $\beta C 93$, previously demonstrated in $\beta C 93 \mathrm{~A}$ and $\gamma \beta \mathrm{C} 93 \mathrm{~A}$ mice (5).

We examined the effects of collateralization on IR-induced mortality. As shown in Figure 1D, no mice died during the reperfusion interval when collateralization was present $(\gamma \beta \mathrm{C} 93, n=3$; $\gamma \beta \mathrm{C} 93 \mathrm{~A}, n=7$; $\beta$ C93A, $n=11)$. However, in the absence of collateralization, whereas only $18 \%$ of $\gamma \beta C 93$ control mice died $(2 / 11)$, mortality was greatly enhanced in $\gamma \beta C 93 \mathrm{~A}(57 \% ; 4 / 7)$ and $\beta C 93 \mathrm{~A}(62 \%$; $8 / 13$ ) mutant mice. Mortality in the absence of collateralization did not differ significantly between $\gamma \beta C 93 \mathrm{~A}$ and $\beta$ C93A mutant mice. Thus, compensatory collateralization protects from IR-induced death even in the absence of $\beta C 93$, but without collateralization, the absence of $\beta C 93$ results in greatly enhanced mortality.

We obtained standard measures of injury of the LV following IR in $\gamma \beta C 93$ and $\gamma \beta C 93 \mathrm{~A}$ mice that survived the 24-hour reper- fusion interval, employing staining with Evans blue and 2,3,5triphenyltetrazolium chloride (TTC): area of recovery, area at risk, and area of necrosis (Figure 1E). In $\gamma \beta C 93 \mathrm{~A}$ versus $\gamma \beta \mathrm{C} 93$ mice in the absence of collateralization, the area of recovery was significantly diminished, and the area of necrosis and the ratio of the area of necrosis to the area at risk was significantly enlarged (whereas the area at risk was not significantly different); notably, enhanced injury was evident in the absence of $\beta C 93$ even when collateralization was present (Figure 1F).

We also assessed cardiac function by echocardiography in $\gamma \beta C 93$ and $\gamma \beta C 93 \mathrm{~A}$ mice that survived the 24-hour reperfusion interval. We measured LV ejection fraction and fractional shortening (Figure $1 G$ ) as well as anterior and posterior LV wall thickening (Figure $1 \mathrm{H})$. All of these measures declined in $\gamma \beta C 93 \mathrm{~A}$ versus $\gamma \beta \mathrm{C} 93$ mice (Figure $1, \mathrm{G}$ and $\mathrm{H}$ ), and significant declines in ejection fraction and fractional shortening were evident in the absence of $\beta C 93$ even when collateralization was present (Figure $1 G$ ). Thus, the absence of $\beta C 93$ induces remodeling of cardiac vasculature and heart and, absent vascular remodeling, greatly increases mortality associated with MI; cardiac injury in the absence of $\beta C 93$ is similarly increased.

We next asked whether protection by SNO-Hb extended to heart failure of nonischemic etiology and in particular to pressure overload-induced cardiomyopathy (11). Compensatory cardiac hypertrophy in response to increased workload is normally accompanied by angiogenesis to maintain oxygenation of hypertrophied cardiomyocytes, and inhibition of angiogenesis leads to heart failure (13). Therefore, it might be predicted that deficient oxygenation resulting from the absence of $\beta \mathrm{C} 93$ would be deleterious.

Pressure overload-induced cardiac hypertrophy was induced by partial occlusion of the aorta (TAC). Two days after TAC, echocardiography revealed significantly greater impairment of $\mathrm{LV}$ function in $\gamma \beta C$ C3A versus $\gamma \beta C 93$ mice across all measures, including decreased ejection fraction (Figure $2 \mathrm{~A}$ ), fractional shortening (Figure 2B), and cardiac output (Figure 2C) as well as increased end systolic diameter ( $\mathrm{LV}$ dilation) (Figure 2D). In addition, the ratios of heart weight (Figure 2E) and lung weight (Figure 2F) to body weight were elevated, indicative of cardiac hypertrophy and pulmonary edema, respectively.

Cohorts of $\gamma \beta C 93, \gamma \beta C 93 \mathrm{~A}$, and $\beta$ C93A mice were followed for 30 days after TAC. Within 5 days after TAC, a single $\gamma \beta C 93$ control animal of 18 mice subjected to TAC died (1/18; 5.6\% mortality), whereas 7 of $11 \gamma \beta C 93 \mathrm{~A}$ mice died (63.6\% mortality) before 
$\gamma \beta \mathrm{C} 93$

A

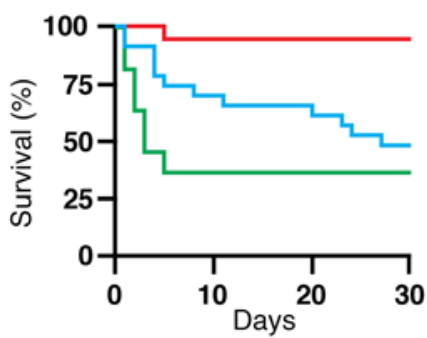

$\gamma \beta$ C93A

B

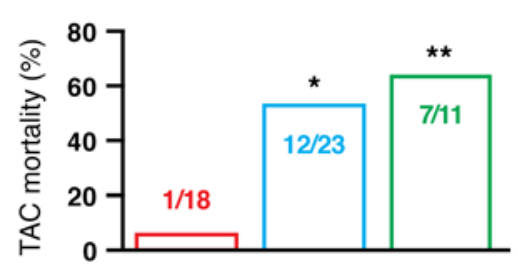

C

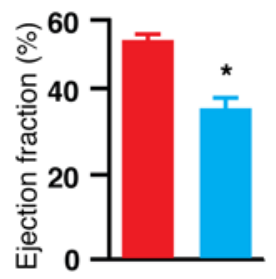

D

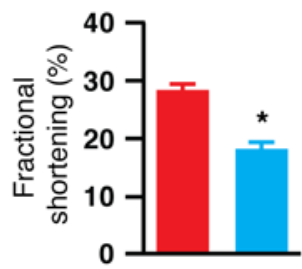

$\mathbf{E}$

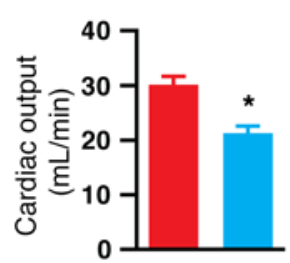

$\mathbf{F}$

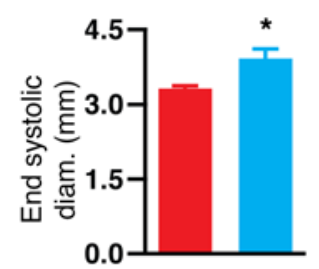

G

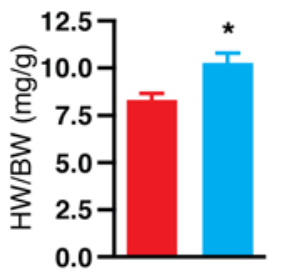

H

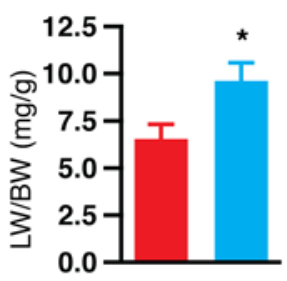

I

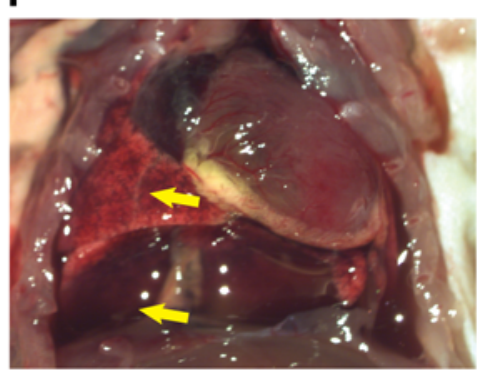

Figure 3. Assessment of chronic effects of pressure overload (TAC) on mortality and injury in C93A mutant animals. Comparisons among $\gamma \beta C 93, \beta C 93 A$, and $\gamma \beta$ C93A mice were made following 4 weeks of TAC. (A) A Kaplan-Meier curve illustrates that, within 5 days after TAC, only 1 of $18 \gamma \beta$ C 93 control animals died ( $5.6 \%$ mortality), whereas 7 of $11 \gamma \beta C 93 A$ mice died (63.6\% mortality) before the population stabilized. Also, 12 of $23 \beta C 93 A$ mice died ( $52.2 \%$ mortality), with deaths accumulating over the full TAC time course. (B) Cumulative mortality over 4 weeks was significantly greater for $\beta$ C93A and $\gamma \beta C 93 A$ versus $\gamma \beta C 93$ mice and did not differ significantly between $\beta$ C93A and $\gamma \beta C 93$ A mice. ${ }^{*} P=0.0019$ vs. $\gamma \beta C 93$ for $\gamma \beta C 93 A$ and ${ }^{* *} P=0.0014$ vs. $\gamma \beta C 93$ for $\gamma \beta C 93 A$ by Fisher's exact test (2-sided). (C-F) Echocardiography in mice surviving the 4-week TAC interval revealed greater impairment of LV function in $\beta$ C93A versus $\gamma \beta C 93$ mice with respect to ejection fraction (C), fractional shortening (D), cardiac output (E), and end systolic diameter (LV dilation) (F). (G and $\mathbf{H})$ Ratios of heart weight with respect to body weight $(\mathbf{C})$ and lung weight with respect to body weight $(\mathbf{H})$ were significantly increased in $\beta$ C93A versus $\gamma \beta C 93$ mice, indicative of increased myocardial hypertrophy and pulmonary edema, respectively. In $\mathbf{C}-\mathbf{H}, n=11$ ( $\gamma \beta C 93)$ or 12 ( $\beta C 93 \mathrm{~A}) ; P<0.01$ by Student's $t$ test (2-tailed). (I) Post-mortem examination revealed lung edema and pleural effusion (yellow arrows; a representative $\beta$ C93A mouse is shown).

the population stabilized (Figures 3, A and B). Similarly, 12 of 23 $\beta C 93 \mathrm{~A}$ mice died (52.2\% mortality), with deaths accumulating over the full post-TAC time course (Figure 3, A and B). Cumulative mortality did not differ significantly between $\gamma \beta C 93 \mathrm{~A}$ and $\beta C 93 \mathrm{~A}$ mice (Figure 3B).

We assessed cardiac function by echocardiography in surviving mice. The small population of survivors precluded meaningful analysis of the $\gamma \beta C 93 \mathrm{~A}$ strain. Echocardiography revealed significantly greater impairment of $\mathrm{LV}$ function in $\beta C 93 \mathrm{~A}$ versus $\gamma \beta C 93$ mice across all measures including ejection fraction (Figure 3C), fractional shortening (Figure 3D), and cardiac output (Figure 3E) as well as significantly increased end systolic diameter (Figure $3 \mathrm{~F}$ ). In addition, $\beta$ C93A mice versus $\gamma \beta \mathrm{C} 93$ mice exhibited significantly increased ratios of heart weight to body weight (Figure 3G) and lung weight to body weight (Figure 3H), again indicative of cardiac hypertrophy and pulmonary edema, respectively. Post-mortem examination confirmed lung edema and pleural effusion in $\beta$ C93A mutant mice (Figure 3I), consistent with clinical heart failure.

Our findings establish a role for $\mathrm{Hb} \beta \mathrm{C} 93$ in ameliorating the consequences of both ischemic and pressure overload-induced cardiac insults and thus demonstrate the central cardiovascular role of RBC-derived SNO-based vasoactivity $(2,3,5)$ in the context of cardiac injury. These findings are consistent with the demonstration that $\beta$ Cys93Ala mutation results in myocardial hypoxia under basal, normoxic conditions (5) by impairing the allosteric mechanism in $\mathrm{Hb}$ through which hypoxia is coupled to compensatory increases in blood flow (2-4). Hypoxia-coupled delivery of SNO-based vasodilatory activity from $\beta C 93$ in vivo would enhance myocardial perfusion following ischemic injury (and thus the area of recovery is diminished and mortality is enhanced following IR in the absence of $\beta$ Cys93) and would support the compensatory myocardial hypertrophy and wall stress induced by TAC (and thus cardiac function is diminished and mortality is enhanced during TAC in the absence of $\beta$ Cys93).

Remarkably, $\beta$ Cys93 is the only residue in $\mathrm{Hb}$ that is conserved across mammals and birds, other than residues that coordinate the $\mathrm{O}_{2}$-carrying heme moiety (7). Our results provide further evidence for the evolutionary selective pressure that underlies this conservation, including adaptive growth of LV collateral circulation to compensate for tissue ischemia. Because deficient delivery of SNO-based vasoactivity by $\mathrm{Hb}$ exacerbates cardiac injury across multiple etiologies, whereas increased RBC-derived NO bioactivity may ameliorate cardiac injury (14), our results suggest the possibility that assessment of SNO-Hb might provide a novel biomarker of cardiac disease. Our findings further suggest that enhancing delivery of SNObased vasodilatory activity from $\mathrm{Hb} \beta \mathrm{Cys} 93$ may represent a potential therapeutic strategy for improving oxygenation in acute coronary syndromes and heart failure and more general- 
ly across multiple pathophysiologies that are characterized by deficient tissue oxygenation.

\section{Methods}

MI model. Male mice at 9 to 16 weeks of age were subjected to IR induced by transient occlusion (30 minutes) of the LCA according to standard procedures (10), described in the Supplemental Methods. Following 24-hour reperfusion, hearts of surviving mice were infiltrated with Evans blue and removed for histological analysis.

TAC model. TAC was carried out as described (15) in male mice 9 to 16 weeks of age. In brief, the aorta was exposed following induction of anesthesia and a ligature of 7-0 silk suture was placed around the aortic arch and an apposed segment of 27-gauge needle, between the right innominate and left carotid arteries. The ligature was tightened, and the needle was removed immediately. The resulting reduction in the cross-sectional area of the arch was approximately $90 \%$.

Echocardiography. Mice were chemically restrained with isoflurane, and transthoracic echocardiography was performed using a Vevo 770 High-Resolution Imaging System equipped with an RMV-707B 30-MHz probe (VisualSonics). Standard M-mode sampling was used through the LV short axis at the midpapillary level. Ejection fraction, fractional shortening, and other parameters were determined using the system's software. LV wall thickening was calculated as follows: (end systolic wall thickness - end diastolic wall thickness)/end diastolic wall thickness $\times 100 \%$.

Statistics. Data are presented as means $( \pm$ SEM) or as ratios with group differences tested using standard parametric methods (i.e. Stu- dent's $t$ test, 2-tailed) or $2 \times 2$ contingency tables with Fisher's exact test as appropriate. In all settings, a $P$ value of less than 0.05 was assumed to indicate a statistically significant difference in the parameter being compared.

Study approval. All experimental procedures were approved by the Institutional Animal Care and Use Committee of Case Western Reserve University School of Medicine and were conducted in accordance with the NIH Guide for the Care and Use of Laboratory Animals (National Academies Press. 2011). All potentially painful procedures were conducted under a surgical plane of anesthesia (typically ketamine/xylazine 100/10 mg/kg), and mice were killed by methods compliant with the American Veterinary Medical Association (AMVA) Guidelines for Euthanasia of Animals (2013 edition).

\section{Author contributions}

RZ, DTH, and JSS designed the studies; RZ conducted the experiments; RZ, DTH, and JDR analyzed the data; all authors participated in writing the manuscript.

\section{Acknowledgments}

This work was supported in part by NIH grants 5P01HL075443 and 1RO1HL126900-01A1. The mouse strains employed were provided by T.M. Townes (University of Alabama, Birmingham, Alabama, USA).

Address correspondence to: Jonathan S. Stamler, Institute for Transformative Molecular Medicine, Case Western Reserve University, 10900 Euclid Avenue, Location Code 7294, Cleveland, Ohio 44106. USA. Phone: 216.368.5725; E-mail: jonathan.stamler@case.edu.
1. Ross JM, Fairchild HM, Weldy J, Guyton AC. Autoregulation of blood flow by oxygen lack. Am JPhysiol. 1962;202:21-24.

2. Stamler JS, et al. Blood flow regulation by Snitrosohemoglobin in the physiological oxygen gradient. Science. 1997;276(5321):2034-2037.

3. Jia L, Bonaventura C, Bonaventura J, Stamler JS. S-nitrosohaemoglobin: a dynamic activity of blood involved in vascular control. Nature. 1996;380(6571):221-226.

4. Singel DJ, Stamler JS. Chemical physiology of blood flow regulation by red blood cells: the role of nitric oxide and S-nitrosohemoglobin. Annu Rev Physiol. 2005;67:99-145.

5. Zhang R, et al. Hemoglobin $\beta$ Cys 93 is essential for cardiovascular function and integrated response to hypoxia. Proc Natl Acad Sci US A. 2015;112(20):6425-6430.

6. Isbell TS, et al. SNO-hemoglobin is not essential for red blood cell-dependent hypoxic vasodila- tion. Nat Med. 2008;14(7):773-777.

7. Nagai K, Perutz MF, Poyart C. Oxygen binding properties of human mutant hemoglobins synthesized in Escherichia coli. Proc Natl Acad Sci US A. 1985;82(21):7252-7255.

8. Parodi O, et al. Myocardial blood flow distribution in patients with ischemic heart disease or dilated cardiomyopathy undergoing heart transplantation. Circulation. 1993;88(2):509-522.

9. Briceno N, Schuster A, Lumley M, Perera D. Ischaemic cardiomyopathy: pathophysiology, assessment and the role of revascularisation. Heart. 2016;102(5):397-406.

10. Michael LH, et al. Myocardial ischemia and reperfusion: a murine model. Am J Physiol. 1995;269(6 Pt 2):H2147-H2154.

11. Rockman HA, et al. Segregation of atrial-specific and inducible expression of an atrial natriuretic factor transgene in an in vivo murine model of cardiac hypertrophy. Proc Natl Acad Sci U S A. 1991;88(18):8277-8281.

12. Gaston B, et al. Essential role of hemoglobin beta-93-cysteine in posthypoxia facilitation of breathing in conscious mice. JAppl Physiol. 2014;116(10):1290-1299.

13. Sano M, et al. p53-induced inhibition of Hif-1 causes cardiac dysfunction during pressure overload. Nature. 2007;446(7134):444-448.

14. Yang J, Gonon AT, Sjöquist PO, Lundberg JO, Pernow J. Arginase regulates red blood cell nitric oxide synthase and export of cardioprotective nitric oxide bioactivity. Proc Natl Acad Sci U S A. 2013;110(37):15049-15054.

15. Hu P, Zhang D, Swenson L, Chakrabarti G, Abel ED, Litwin SE. Minimally invasive aortic banding in mice: effects of altered cardiomyocyte insulin signaling during pressure overload. Am J Physiol Heart Circ Physiol. 2003;285(3):H1261-H1269. 